\title{
Radio measurements of constant variation, and perspectives with ALMA
}

\author{
Françoise Combes \\ LERMA, Observatoire de Paris, 61 Av. de l'Observatoire, F-75014, Paris \\ email: francoise.combes@obspm.fr
}

\begin{abstract}
In the radio domain, absorption lines in front of quasars of $\mathrm{CO}, \mathrm{HI}, \mathrm{OH}, \mathrm{HCO}^{+}$, $\mathrm{HCN}$, up to $\mathrm{NH}_{3}$ and $\mathrm{CII}$ are providing interesting constraints on fundamental constant variation $(\alpha$ and $\mu$ ). With more absorbing systems, and a wider redshift range, they could be more competitive than optical studies. This could come with ALMA, with more than one order of magnitude in sensitivity.
\end{abstract}

Up to now, at intermediate and high redshift, between $\mathrm{z}=0.25$ to $\mathrm{z}=0.89$, only four absorption lines systems have been detected in the millimeter range and a fifth system at 0.765 , at the $\mathrm{OH}$ $18 \mathrm{~cm}$ lines (Kanekar et al. 2005). Out of these 5 systems, 3 are intervening lensing galaxies (and the background quasar is multiply imaged), and 2 correspond to an absorption of the host (PKS1413+135, B3-1504+377, for an overview see Combes \& Wiklind 1996; Wiklind \& Combes 1994 to 1998).

A global comparison of all molecular lines observed with the HI- $21 \mathrm{~cm}$ absorption lines in PKS1413 and B0218 systems, the two narrowest line systems, have given quite stringent constraints on $y=\alpha^{2} g_{p} \mu, \Delta y / y=(-0.20 \pm 0.44) 10^{-5}$ and $\Delta y / y=(-0.16 \pm 0.54) 10^{-5}$ respectively (Murphy et al. 2001). The precision is comparable to the MM method (Murphy et al. 2003), with a limited number of absorbing systems.

The high sensitivity if the $\mathrm{NH}_{3}$ inversion lines to variation in the $\mu$ ratio (Flambaum \& Kozlov 2007) was used by Henkel et al. (2009) in a recent multi-line study of PKS1830 at $z \sim 0.9$, and Murphy et al. (2008) for B0218 at $z \sim 0.7$. They find a limit of $\Delta \mu / \mu<1.410^{-6}$ and $\Delta \mu / \mu<1.810^{-6}$ respectively.

Clearly, the radio method suffers from the rarity of the objects, and the fact that they have not yet been discovered at high redshift. The main caveats are that the lines compared come from different molecules, which might have intrinsic velocity offsets, due to several reasons, chemistry, excitation, temperature, density etc... When very different frequencies are compared (HI to CO for instance), the background continuum source has different sizes, and the absorbing medium is not the same. Also, the continuum source varies in both intensity and shape, and the compared lines are not always observed simultaneously. Only large statistics could smooth the errors down.

On the positive side, the radio domain is favoured by the high spectral resolution and the very narrow lines due to cold gas, the exquisite precision of the frequency calibration, and the well-known rest frequencies. Also, the sensitivity of the line position to the variation of constants is much higher (by a factor 100 for $\mathrm{NH}_{3}$ ).

Fortunately, the sensitivity of ALMA will be able to detect many more continuum sources, to search for absorption lines, and at larger redshifts. ALMA will have a much wider bandwidth, allowing the search of absorption, even if not previously detected in the optical or HI- $21 \mathrm{~cm}$. The redshift will be obtained directly in the millimeter.

It is possible to predict the number of continuum sources that can be selected as targets for absorption searches with ALMA. The density of flat-spectrum quasars has been shown to follow the same curve as optical quasars, a curve peaking at $z \sim 2$, very similar to the star formation history (Wall et al. 2005). They are still of significant density at $z \sim 3$.

Keywords. Galaxies: ISM - Quasars: absorption lines - Cosmology: observations 